\title{
Porcelanas elétricas aluminosas: Parte I - Revisão da literatura
}

\section{(Aluminous electrical porcelain: Part I - Literature review)}

\author{
A. L. Chinelatto ${ }^{1}$, D. P. F. de Souza ${ }^{2}$ \\ ${ }^{1}$ Departamento de Engenharia de Materiais, Universidade Estadual de Ponta Grossa \\ Av. Carlos Cavalcanti 4748, Ponta Grossa, PR, 84030-900 \\ ${ }^{2}$ Departamento de Engenharia de Materiais, Universidade Federal de S. Carlos \\ Rod. Washington Luiz km 235, C.P. 676, S. Carlos, SP, 13565-905 \\ adilson@uepg.br,dulcina@power.ufscar.br
}

\begin{abstract}
Resumo
Porcelanas são cerâmicas multifásicas produzidas a partir de matérias-primas naturais. $\mathrm{O}$ desenvolvimento da microestrutura dessas cerâmicas é dependente da composição, das características das matérias-primas e do processamento cerâmico. Devido à complexidade da porcelana, várias aspectos relacionados com a evolução da microestrutura e os seus efeitos nas propriedades não são ainda bem entendidos, apesar do grande número de publicações sobre este assunto. A parte I deste artigo é uma revisão da literatura sobre porcelanas aluminosas e enfoca o efeito das composições nas propriedades elétricas e mecânicas, como também a influência de alguns aditivos no desenvolvimento da microestrutura.
\end{abstract}

Palavras-chave: porcelana elétrica aluminosa, composições, microestrutura, propriedades.

\begin{abstract}
Porcelains are multiphase ceramics produced from natural raw materials. The microstructural development of these ceramics depends on their composition, on the characteristics of the raw materials, and on the ceramic processing. Due to the complexity of porcelains, several aspects of the microstructural evolution and its effects on the properties of porcelains are as yet little understood, despite the large number of reports published on the subject. Part I of this article is a literature review on aluminous porcelains, focusing on the effect of their compositions on their electrical and mechanical properties, as well as the influence of some additives on their microstructural development.
\end{abstract}

Keywords: aluminous electrical porcelain, compositions, microstructure, properties.

\section{INTRODUÇÃO}

Porcelanas são cerâmicas produzidas com matérias-primas naturais e utilizadas na fabricação de uma grande variedade de produtos cerâmicos apesar de ser considerada a mais complexa de todas as cerâmicas.

Nestas cerâmicas, as ligações iônicas com forte caráter covalente restringem a mobilidade dos elétrons e as tornam bons isolantes elétricos e, por consequiência, possui um grande número de aplicação na indústria eletroeletrônica. Entre os vários tipos de porcelanas, as mais utilizadas como isoladores elétricos são as porcelanas triaxiais compostas por quartzo, feldspato e argila contudo, a substituição total ou parcial do quartzo por alumina tem sido utilizada para promover aumento da resistência mecânica [1].

As porcelanas formadas por alumina, feldspato e caulim são chamadas de porcelanas aluminosas e são utilizadas na fabricação de isoladores elétricos de alta tensão, por possuírem propriedades dielétricas adequadas e propriedades mecânicas superiores às das porcelanas que utilizam o quartzo. Estas propriedades dielétricas e mecânicas são dependentes da microestrutura desenvolvida durante o processamento cerâmico. A presença de impurezas, a temperatura, o tempo e a atmosfera de sinterização influenciam fortemente as reações químicas e o desenvolvimento microestrutural da porcelana. Desta maneira, é necessário o entendimento da relação entre essas variáveis para desenvolver produtos de baixo custo e com boas propriedades mecânicas e dielétricas.

Apesar da porcelana ser conhecida há séculos e ter sido extensivamente estudada durante a primeira metade do século XX, a busca do entendimento do desenvolvimento microestrutural ganhou novo impulso com o avanço de técnicas de análise de microestrutura, especificamente Microscopia Eletrônica de Varredura (MEV) e Transmissão (MET) associadas com Microanálise por Energia Dispersiva (EDS). Outro fator que também contribuiu para o interesse recente nesse assunto foi o avanço das pesquisas na área de vidros, visto que esta fase ocupa grande fração volumétrica da porcelana.

Na parte I deste artigo é apresentada uma revisão da literatura enfocando a relação entre composição, microestrutura e propriedades como também o efeito de aditivos no desenvolvimento da microestrutura. Na parte II serão mostrados resultados de 3 composições de porcelana aluminosas preparadas com matérias-primas nacionais. 


\section{DESENVOLVIMENTO MICROESTRUTURAL DU- RANTE A SINTERIZAÇÃO DE PORCELANAS TRIAXIAIS}

As matérias-primas utilizadas na fabricação de porcelanas triaxiais são: a argila, o feldspato e o quartzo ou a alumina. A elevada área superficial da argila combinada com a morfologia de suas partículas, placas finas, fornece plasticidade à massa. A argila deve ser caulinítica, a fim de fornecer a caulinita $\left(\mathrm{Al}_{2} \mathrm{O}_{3} \cdot 2 \mathrm{SiO}_{2} \cdot 2 \mathrm{H}_{2} \mathrm{O}\right)$, que durante a sinterização irá se decompor e formar cristais de mulita, chamados de mulita primária. Muitas composições utilizam o caulim ao invés da argila como fonte de caulinita, entretanto os caulins não fornecem plasticidade à massa, sendo necessária a adição de pequenas quantidades de argila plástica para conferir trabalhabilidade à massa e resistência mecânica a verde.

A função do feldspato na composição das porcelanas triaxiais é atuar como fundente viabilizando a sinterização via fase líquida. Os feldspatos normalmente usados em cerâmicas são os feldspatos potássicos $\left(\mathrm{K}_{2} \mathrm{O} \cdot \mathrm{Al}_{2} \mathrm{O}_{3} \cdot 6 \mathrm{SiO}_{2}\right)$ e os feldspatos sódicos $\left(\mathrm{Na}_{2} \mathrm{O} \cdot \mathrm{Al}_{2} \mathrm{O}_{3} \cdot 6 \mathrm{SiO}_{2}\right)$. $\mathrm{O}$ feldspato potássico historicamente é o fundente mais utilizado nas porcelanas pois ao fundir gera um líquido com viscosidade maior que o feldspato sódico [2].

O quartzo, responsável pela formação do esqueleto da peça de porcelana, participa na formação da microestrutura de várias formas. Por ser a fração mais grosseira das partículas na composição da porcelana diminui a retração durante a secagem evitando assim o trincamento. Durante quase todo o processo de queima a reatividade do quartzo permanece baixa, ajudando assim a prevenir a deformação do corpo. Em temperaturas mais elevadas o quartzo se dissolve no vidro feldspáltico aumentando a quantidade de silício no vidro e mantendo a viscosidade elevada, ajudando a evitar a deformação piroplástica. No resfriamento, o quartzo não dissolvido durante a sinterização pode deteriorar a resistência mecânica devido à expansão volumétrica que ocorre durante a mudança de fase $\beta \rightarrow \alpha$. Neste aspecto, a substituição do quartzo por alumina é vantajoso por não apresentar transição de fase no resfriamento e assim não comprometer a resistência mecânica da peça [1, 3]. Entretanto o preço significativamente elevado da alumina comparada ao do quartzo e o aumento na temperatura de sinterização, implica em aumento significativo dos custos da porcelana e se torna a principal desvantagem de seu uso [1]. A utilização da bauxita ao invés da alumina, tem sido considerada como uma alternativa para diminuir os custos de produção [4, 5].

Durante a sinterização das porcelanas triaxiais ocorrem diversas reações químicas, as quais são afetadas pela temperatura, tempo e atmosfera do forno. Nessas reações, o equilíbrio muitas vezes não é atingido devido ao longo tempo necessário para que isto ocorra. As principais reações do processo de sinterização das porcelanas triaxiais são [1, 6-9]:

- Transformação da caulinita $\left(\mathrm{Al}_{2} \mathrm{O}_{3} \cdot 2 \mathrm{SiO}_{2} \cdot 2 \mathrm{H}_{2} \mathrm{O}\right) \mathrm{em}$ metacaulinita $\left(\mathrm{Al}_{2} \mathrm{O}_{3} \cdot 2 \mathrm{SiO}_{2}\right)$, que ocorre aproximadamente a $550^{\circ} \mathrm{C}$. Esta reação é endotérmica e é acompanhada pela reorganização do alumínio octaedricamente coordenado para um alumínio tetraedricamente coordenado na metacaulinita [10].

- Transformação do quartzo $\alpha \rightarrow \beta$ a $573^{\circ} \mathrm{C}$.

- Transformação da metacaulinita $\left(\mathrm{Al}_{2} \mathrm{O}_{3} \cdot 2 \mathrm{SiO}_{2}\right)$ para uma estrutura tipo espinélio e uma fase amorfa de sílica livre, a qual ocorre a $\sim 980{ }^{\circ} \mathrm{C}$. A exata estrutura da fase espinélio ainda não está bem definida e apresenta controvérsias na literatura. Alguns autores [10-12] acreditam ser uma fase espinélio contendo silício e alumínio e outros [13, 14], acreditam ser alumina- $\gamma$ contendo silício.

- Início da formação da fase líquida ao se atingir a temperatura eutética $\left(\sim 1050{ }^{\circ} \mathrm{C}\right.$ para o feldspato sódico e $\sim 990$ ${ }^{\circ} \mathrm{C}$ para o feldspato potássico). A composição do eutético formado no contato do feldspato potássico com a argila é dada por $9,5 \%$ de $\mathrm{K}_{2} \mathrm{O}, 10,9 \%$ de $\mathrm{Al}_{2} \mathrm{O}_{3}$ e $79,9 \%$ de $\mathrm{SiO}_{2}$.

- Formação de mulita $\left(3 \mathrm{Al}_{2} \mathrm{O}_{3} \cdot 2 \mathrm{SiO}_{2}\right) \mathrm{a} \sim 1075^{\circ} \mathrm{C}$ a partir da fase espinélio. Em vidros de $\mathrm{Al}_{2} \mathrm{O}_{3}-\mathrm{SiO}_{2}$ a cristalização da mulita ocorre a partir de uma região rica em $\mathrm{Al}_{2} \mathrm{O}_{3}$ decorrente de um processo de separação de fase $[15,16]$. Como a exata estrutura da fase espinélio ainda não é bem estabelecida, existe dificuldade no entendimento do mecanismo de formação da mulita a partir desta fase $[11,12]$.

- Dissolução do quartzo entre $\sim 990^{\circ} \mathrm{C}$ e $1200^{\circ} \mathrm{C}$, quando a fase líquida torna-se saturada com silício e começa então a transformação do quartzo em cristobalita. Nas porcelanas triaxiais aluminosas o quartzo residual proveniente das matériasprimas sofre dissolução acima de $1100{ }^{\circ} \mathrm{C}$, praticamente desaparecendo em temperaturas acima de $1250^{\circ} \mathrm{C}$ [17].

- A alumina permanece inerte durante a sinterização, tendo uma taxa de dissolução no vidro feldspáltico muito baixa até temperaturas de $1450{ }^{\circ} \mathrm{C}[16,1]$.

- Transformação do quartzo $\beta \rightarrow \alpha$, durante o resfriamento $\left(\sim 573{ }^{\circ} \mathrm{C}\right)$, resultando em uma diminuição de $2 \%$ no volume dos grãos de quartzo, produzindo tensões suficientes para trincar a matriz ou o próprio grão.

A microestrutura da porcelana triaxial após a sinterização consiste em grãos grosseiros de quartzo ou alumina misturados a finos cristais de mulita mantidos juntos por uma matriz vítrea. Os cristais de mulita possuem diversas origens as quais são responsáveis pelos nomes atribuídos a elas. A mulita formada pela seqüência de reações caulinita-mulita, é chamada de mulita primária. Esta mulita aparece na forma de aglomerados de pequenos cristais com tamanho menor que $0,5 \mu \mathrm{m}$ [18] e recebe este nome devido ao fato de se formar a baixa temperatura [1]. Um outro tipo de mulita, chamada mulita secundária, é formada a partir da nucleação e crescimento de cristais na região do líquido feldspáltico e se apresenta na forma de longos cristais $(>1 \mu \mathrm{m})$ [18]. Iqbal e Lee [19] identificaram ainda a presença de um outro tipo de mulita secundária mais alongada e propuseram a seguinte nomenclatura para os diversos tipos de mulita: a mulita primária foi chamada de tipo-I, as mulitas secundárias foram chamadas de tipo-II (a menor) e tipo-III (a mais alongada). Carty [1] faz referência também a um outro tipo de mulita, que aparece em porcelanas aluminosas. Esta mulita aparece ao redor dos grãos de alumina, devido à dissolução destes grãos no líquido feldspáltico. Iqbal e Lee [18] denominaram esta mulita de mulita terciária.

De acordo com Iqbal e Lee [18], durante a sinterização da 
porcelana triaxial a mulita secundária é formada a partir da superfície da argila caulinítica e cresce em direção ao líquido originado do feldspato. O líquido proveniente do feldspato é mais fluido que o proveniente da argila, pois possui uma alta quantidade de íons alcalinos e alcalinos terrosos e, como consequiência, os cristais de mulita secundária são maiores que os da mulita primária $[18,20]$. A composição da mulita muda de acordo com a origem [18]. A mulita primária formada na região rica em argila contém mais alumina $\left(2 \mathrm{Al}_{2} \mathrm{O}_{3}: 1 \mathrm{SiO}_{2}\right)$ que a mulita secundária (tipo-II e tipo-III) cristalizada na região do feldspato $\left(3 \mathrm{Al}_{2} \mathrm{O}_{3}: 2 \mathrm{SiO}_{2}\right)$. $\mathrm{O}$ tamanho e a forma dos cristais de mulita são controlados pela viscosidade do líquido onde eles se formam e crescem. A viscosidade do líquido por sua vez é controlada por sua composição e temperatura [20].

A Fig. 1 mostra uma série de micrografias obtidas por microscopia eletrônica de varredura que ilustram como as fases descritas acima coexistem na porcelana aluminosa [17].

\section{COMPOSIÇÃO DAS PORCELANAS (\% EM PESO)}

Uma larga faixa de composição para porcelanas é encontrada na literatura, variando desde porcelanas ricas em quartzo (silicosas) até porcelanas ricas em alumina (aluminosas). Para utilização em isoladores de baixa tensão, Buchanan [21] apresenta uma faixa de composição para porcelanas silicosas, onde a quantidade de argila caulinítica varia entre $40 \%$ e $60 \%$, a quantidade de feldspato varia entre $20 \%$ e $35 \%$ e a quantidade de quartzo entre $20 \%$ e $30 \%$. Estas composições são sinterizadas entre $1250{ }^{\circ} \mathrm{C}$ e $1330{ }^{\circ} \mathrm{e}$ apresentam constante dielétrica entre 5,5 e 7 e tg $\delta$ de 0,005 .

Norton [22] sugere para isoladores de alta tensão porcelanas aluminosas, com $15 \%$ de caulim, $20 \%$ de argila, $25 \%$ de feldspato e $40 \%$ de alumina, para uma porcelana rica em alumina, e $20 \%$ de caulim, $25 \%$ de argila, $35 \%$ de feldspato e $20 \%$ de alumina para uma porcelana com baixo teor de alumina.

Biesen et al [23] utilizaram aluminas com diferentes purezas e tamanho de partículas para preparar porcelanas com a composição $30 \%$ de alumina, $45 \%$ de caulim, $17 \%$ de quartzo e $8 \%$ de feldspato e mostraram que a trabalhabilidade da massa é fortemente influenciada pelas características da alumina.

As propriedades mecânicas e elétricas de porcelanas silicosas dependem da relação Feldspato/Quartzo. Bishai et al [24] determinaram que quando esta relação é igual a 0,85 melhores propriedades mecânicas e elétricas são obtidas.

Goulart et al [25] estudaram porcelanas silicosas (22\% de quartzo, $28 \%$ de feldspato, $18 \%$ de caulim e $32 \%$ de argila) e também porcelanas aluminosas ( $22 \%$ a $40 \%$ de alumina, $21,6 \%$ a $28 \%$ de feldspato, $13,8 \%$ a $18 \%$ de caulim e $24,6 \%$ a $32 \%$ de argila) e observaram que as porcelanas ricas em alumina, possuem tensão de ruptura sob flexão maior que as ricas em quartzo, em todas as temperaturas de sinterização estudadas. Este comportamento foi confirmado posteriormente por Kobayashi et al. [26] que estudaram várias composições de porcelanas aluminosas, com o teor de alumina variando de 0 a $50 \%$. A tensão de ruptura foi de $200 \mathrm{MPa}$ para amostras sem a presença de alumina e de $371 \mathrm{MPa}$ para amostras com 50\% de alumina.

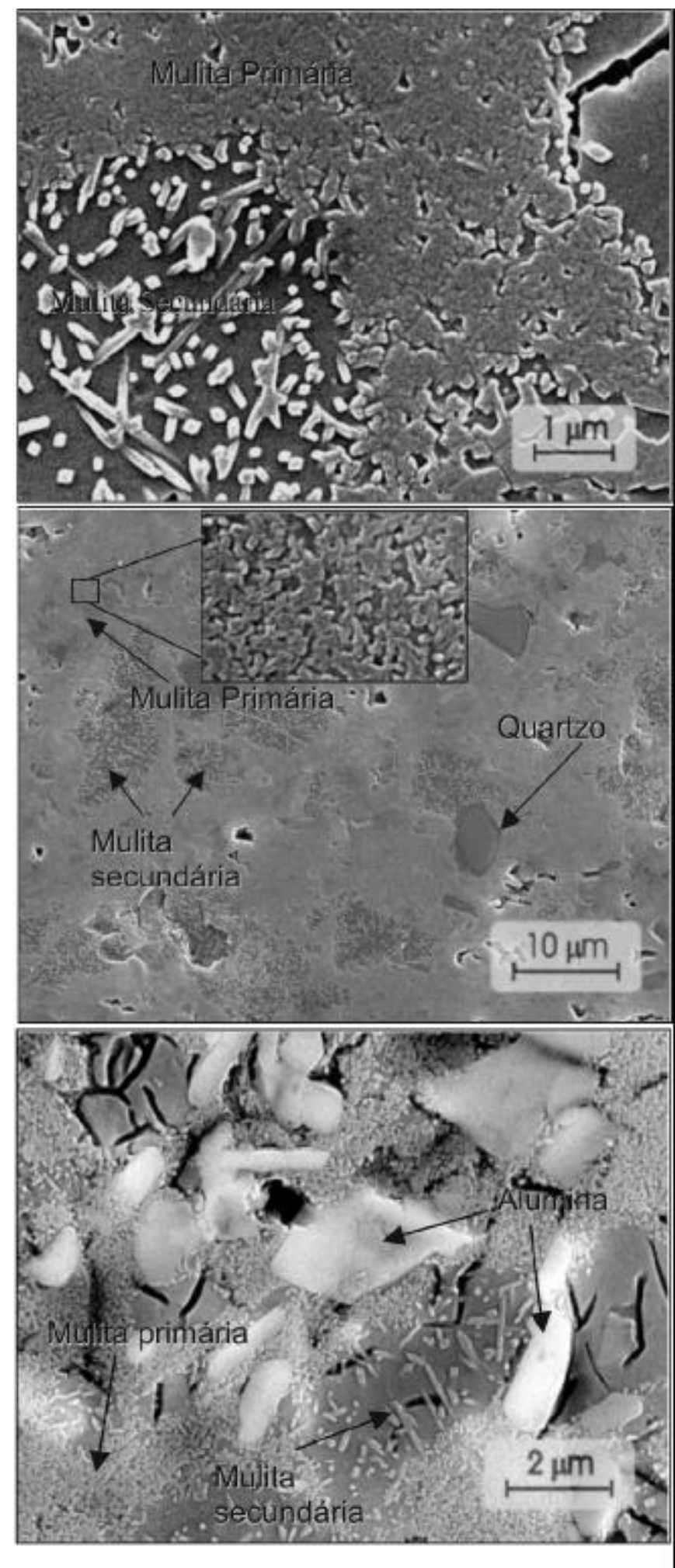

Figura 1: Micrografias obtidas por MEV mostrando as fases cristalinas presentes em uma porcelana triaxial aluminosa. [Figure 1: SEM micrographs showing the crystalline phases present on the triaxial aluminous porcelain.]

Popova e Kreimer [27] mostraram que na composição de porcelanas aluminosas, um aumento de $\mathrm{K}_{2} \mathrm{O}$ em relação a $\mathrm{Na}_{2} \mathrm{O}$ ou de $\mathrm{Al}_{2} \mathrm{O}_{3}$ em relação a quantidade de $\left(\mathrm{K}_{2} \mathrm{O}+\mathrm{Na}_{2} \mathrm{O}\right)$ aumenta a temperatura de sinterização. Este resultado ilustra com 
clareza que a origem da matéria prima natural, do ponto de vista geográfico, é determinante no desenvolvimento da microestrutura visto que a composição química e mineralógica das matérias-primas naturais são peculiares de cada região.

Outros trabalhos são encontrados na literatura [28-30] onde uma ou mais das matérias-primas são substituídas por outras, gerando uma grande diversidade de composições. As substituições visam redução de custos ou melhoria de propriedades. Contudo, as propriedades não são determinadas apenas pela composição em termos de matérias-primas mas também pela composição mineralógica de cada matéria-prima utilizada.

\section{PROPRIEDADES MECÂNICAS DAS PORCELANAS}

A resistência mecânica das porcelanas pode ser drasticamente afetada pela transição de fase das partículas de quartzo que estão presentes mesmo nas porcelanas aluminosas, introduzidas via argila, feldspato e caulim. A busca pela melhoria dessa propriedade resultou no desenvolvimento de teorias que tentam relacionar a microestrutura com o desempenho mecânico. As três principais teorias, muito bem resumidas por Carty e Senapati [1] são:

Hipótese do Reforço da Matriz: Nesta teoria a diferença de coeficiente de expansão térmica entre a matriz (fase vítrea) e os grãos dispersos, tais como quartzo e alumina ou outras fases cristalinas como mulita e cristobalita, produzem forte tensão compressiva na fase vítrea. Esta tensão térmica induzida leva a um aumento da resistência mecânica da porcelana.

Para uma partícula esférica em uma matriz isotrópica, a diferença entre os coeficientes de expansão térmica podem levar à tensões radiais ou tangenciais. A tensão total, $\mathrm{P}$, na partícula pode ser dada por:

$$
\mathrm{P}=\frac{\Delta \alpha \Delta \mathrm{t}}{\frac{1+\mathrm{v}_{\mathrm{m}}}{2 \mathrm{E}_{m}}+\frac{1+2 v_{\mathrm{p}}}{\mathrm{E}_{\mathrm{p}}}}
$$

onde $\Delta \alpha$ é a diferença no coeficiente de expansão térmica entre a matriz e a partícula; $\Delta \mathrm{T}$ é a diferença de temperatura do sistema matriz-partícula no resfriamento; $v_{m}, v_{p}, E_{m}$ e $E_{p}$ são os módulos de Poisson e os módulos elásticos da matriz e da partícula respectivamente.

A natureza das trincas nas peças é dependente dos coeficientes de expansão térmica das fases presentes. Se as partículas contraem mais que a matriz, $\mathrm{P}$ é negativo resultando em um trincamento circunferencial ao redor das partículas. Este é o caso para partículas de quartzo e matriz vítrea de natureza feldspáltica. A tensão e o trincamento gerados devido a presença de partículas de quartzo, tendem a ser mais severos devido a rápida transformação do quartzo $\beta \rightarrow \alpha$ durante o resfriamento. O tamanho da partícula de quartzo é crucial no desenvolvimento das trincas, pois partículas muito pequenas não são suficientes para colocar a matriz sob tensão, enquanto que partículas muito grandes podem gerar fraturas interconectadas levando a um empobrecimento das propriedades mecânicas. Foi determinado que o tamanho ideal das partículas de quartzo é $25 \mu \mathrm{m}$ [31].

Hipótese da Mulita: Nesta teoria, uma das mais antigas sobre a resistência mecânica das porcelanas e não leva em consideração os efeitos da transição de fase das partículas de quartzo, estabelece que ela depende unicamente do entrelaçamento de finos cristais de mulita em forma de agulhas sendo que a resistência mecânica aumenta com o aumento da quantidade de mulita [32-34]. O crescimento dos cristais de mulita, com conseqüente redução de seu número, reduz o entrelaçamento dos cristais, que é muito eficiente com cristais menores, resultando numa diminuição da resistência mecânica. O tamanho dos cristais de mulita pode ser controlado pela temperatura de sinterização. A mulita secundária, por ter cristais com morfologia acicular e agulhas de pequenos diâmetros, poderá aumentar mais a resistência mecânica que a mulita primária, desde que os cristais se mantenham devidamente entrelaçados.

Hipótese de Reforço por Dispersão de Partículas: Esta teoria propõe que partículas dispersas na matriz vítrea, com tamanho próximo ao tamanho das falhas de Griffith, levam a um aumento da resistência mecânica. Nesse caso, a resistência mecânica é dependente da fração volumétrica e do tamanho das partículas dispersas na matriz, para alta fração volumétrica, e é dependente apenas da fração volumétrica para baixa fração volumétrica.

Como em corpos de porcelana os coeficientes de expansão térmica da matriz raramente são idênticos aos das partículas dispersas existe sempre um efeito de reforço da matriz. Além disso, o entrelaçamento dos cristais de mulita sempre existe, contribuindo para o aumento da resistência mecânica. Portanto, uma teoria geral sobre a relação entre microestrutura e propriedade mecânica de porcelana deveria levar em consideração os três mecanismos descritos acima. Experimentalmente é observado que a resistência mecânica de uma peça de porcelana é resultado do efeito concomitante de todas as variáveis abordadas acima, isto é: da quantidade e tamanho das agulhas de mulita, tamanho e forma das partículas de quartzo e/ou alumina dispersas, diferença do coeficiente de expansão da fase vítrea e cristalinas, e propriedade mecânica característica da fase vítrea. Maslennikova et. al. [35] verificaram que a resistência mecânica de porcelanas sem a adição de quartzo ou de alumina aumenta com a diminuição da quantidade de feldspato. Este aumento foi atribuído à quantidade de mulita e à mudança na composição da fase vítrea resultando em uma fase com melhor resistência mecânica devido à diminuição da quantidade de óxidos de metais alcalinos com conseqüente aumento das ligações $\mathrm{Si}-\mathrm{O}-\mathrm{Si}$.

Sugiyama et. al. [36] observaram um aumento do módulo de ruptura quando alumina foi introduzida na porcelana silicosa e quando o quartzo foi substituído por alumina nessas porcelanas. A melhoria do módulo de ruptura foi atribuída não apenas ao aumento do módulo elástico causado pela presença de alumina- $\alpha$, mas também devido à diminuição no tamanho 
Tabela I - Valores do máximo módulo de ruptura (MOR) de porcelanas de quartzo e porcelanas aluminosas obtidos por vários autores.

[Table I - Values of maximum bending strength of quartz porcelain and aluminous porcelain obtained by several authors.]

\begin{tabular}{ccc}
\hline Referência & $\begin{array}{c}\text { Tipo de } \\
\text { porcelana }\end{array}$ & MOR (MPa) \\
\hline$[23]$ & Quartzo+Alumina & 84 \\
\hline$[29]$ & Quartzo & 130 \\
\hline$[49]$ & Alumina & 140 \\
& Quartzo & 95 \\
\hline$[38]$ & Alumina & 150 \\
\hline$[25]$ & Quartzo+Alumina & 150 \\
& Alumina & 196 \\
\hline$[36]$ & Quartzo+Alumina & 150 \\
\hline$[35]$ & -------- & 152 \\
\hline$[37]$ & Alumina & 215 \\
\hline$[28]$ & Alumina & 257 \\
\hline$[26]$ & Alumina & 371 \\
\hline$[45]$ & Alumina & 114 \\
\hline$[46]$ & Alumina & 240 \\
\hline$[47]$ & Alumina & 242 \\
\hline
\end{tabular}

dos defeitos causados pela presença das pequenas partículas (alumina- $\alpha$ ) dispersas na fase vítrea. Este resultado concorda com Orlova et. al. [37] que mostraram que o tamanho e a forma dos cristais de alumina tem significante efeito nas propriedades das porcelanas e sugerem que para se obter porcelanas com bom desempenho mecânico, o tamanho dos grãos de alumina não deve exceder $3 \mu \mathrm{m}$ e devem estar completamente dispersos.

Kreimer e Chistyakova [38] mostraram que a resistência mecânica aumenta com o aumento das fases cristalinas e com a diminuição da fase vítrea sendo que o maior aumento ocorre quando a quantidade de mulita é aumentada e a quantidade de quartzo residual e de fase vítrea são diminuídas. Esses resultados foram confirmados por Maiti e Kumar [29] que substituíram o quartzo e feldspato por silimanita e vidros cristalizados e observaram aumento de $70 \%$ na resistência mecânica das composições estudadas. Este aumento foi atribuído à redução na quantidade de fase vítrea com conseqüente aumento da fase cristalina.

De acordo com Liebermann $[39,40]$ é vantajoso utilizar alumina ao invés de quartzo em isoladores devido ao significante aumento da resistência mecânica. Esta melhoria é atribuída ao fato de que, em um sistema multifásico, a introdução de um componente com alto módulo elástico, eleva o módulo elástico do sistema e, por conseqüência, a resistência mecânica. Liebermann também observou que o tamanho de grão e a dispersão dos grãos de alumina na porcelana também influenciam o comportamento mecânico como já observado por Orlova et. al. [37]. Liebermann [40] sugeriu vários procedimentos para aumentar a resistência de porcelanas aluminosas. Estas sugestões, em ordem de prioridade, são:

1. desenvolver microestruturas livres de quartzo residual, proveniente das matérias-primas pois, dependendo do tamanho do quartzo, ele pode gerar trincas,

2. formar a fase vítrea prematuramente,

3. aumentar a quantidade de alumina, utilizando o máximo na forma de alumina- $\alpha$, devido ao seu alto módulo elástico e a sua alta densidade,

4. utilizar atmosfera de sinterização redutora acima de $1000^{\circ} \mathrm{C}$ e utilizar a mínima temperatura de sinterização,

5. fazer resfriamento rápido após a sinterização.

Ainda de acordo com Liebermann [40], para se obter microestrutura mecanicamente forte e estável, o teor de mulita deve ser menor ou igual $15 \%$, o de alumina- $\alpha$ maior ou igual a $40 \%$, o de fase vítrea de aproximadamente $45 \%$ e o de quartzo residual menor que $1 \%$. A microestrutura deve estar livre de heterogeneidades com máxima quantidade de fase cristalina interconectada.

A Tabela I mostra valores de módulo de ruptura, obtidos por flexão em três pontos para porcelanas de quartzo e porcelanas aluminosas. A grande variação nos valores de módulo de ruptura encontrados pelos diversos autores é devida principalmente à variação de composição entre as diversas porcelanas analisadas.

\section{PROPRIEDADES DIELÉTRICAS DAS PORCELANAS}

Um isolador elétrico é caracterizado por possuir elevada resistividade elétrica, elevada rigidez dielétrica, baixo fator de dissipação e baixa constante dielétrica. A elevada resistividade elétrica previne a passagem de corrente elétrica através do isolador; a rigidez dielétrica, definida como a máxima diferença de potencial que o dielétrico suporta sem que ocorra uma descarga elétrica, deve ser elevada para evitar o colapso catastrófico do isolador em alta voltagem; o fator de dissipação, que mede a razão entre a parte irrecuperável e recuperável da energia elétrica quando um campo elétrico é aplicado no interior do material sendo que a energia irrecuperável é consumida na forma de calor, deve ser baixo para que o isolador não aqueça em uso; a baixa constante dielétrica evita polarização de modo a não ocorrer armazenamento localizado de cargas no isolante. Valores típicos das propriedades dielétricas para que um material possa ser considerado um bom isolador elétrico é apresentado na Tabela II.

A literatura é discrepante quando aborda a relação entre propriedades dielétricas e microestrutura de porcelanas. Tadzhiev et. al. [41], Maslennikova et. al. [35] e Bishai et. al. [24] determinaram que o fator de dissipação diminui com o aumento da quantidade de mulita e diminuição da fase vítrea. Por outro lado Chaudhuri et. al. [42] mostraram que o aumento na quantidade ou no tamanho dos cristais mulita diminui a resistividade elétrica da porcelana, e que o aumento da quantidade de quartzo, cristobalita ou fase vítrea aumenta a resistividade. $\mathrm{O}$ aumento da condutividade elétrica associado ao aumento da quantidade de mulita foi atribuído tanto à presença de vacâncias de oxigênio na mulita, que a transforma num condutor iônico, 
Tabela II - Valores típicos para um bom isolador elétrico [21]. [Table II - Typical values for a good electrical isolator.]

\begin{tabular}{ll}
\hline Constante dielétrica $(\kappa)$ & $\leq 30$ \\
\hline Resistividade elétrica $(\rho)$ & $\geq 10^{14}(\Omega . \mathrm{m})$ \\
\hline Fator dissipação $(\operatorname{tg} \delta)$ & $\leq 0,001$ \\
\hline Rigidez dielétrica & $\geq 5,0 \mathrm{kV} / \mathrm{mm}$ \\
\hline
\end{tabular}

como à substituição de íons $\mathrm{Al}^{3+}$ por íons de metais de transição, o que gera um comportamento semicondutor.

Com relação à constante dielétrica, Bishai et. al. [24] determinaram que ocorre aumento com o aumento da fase vítrea, enquanto que Chaudhuri e Sarkar [43] mostraram que a constante dielétrica apresenta um comportamento parabólico em função da quantidade de cada uma das fases da porcelana (mulita, quartzo, fase vítrea e cristobalita). $O$ valor da constante dielétrica aumenta até um valor máximo depois cai com o aumento da quantidade de mulita e quartzo. Já para as fases cristobalita e fase vítrea, o valor da constante dielétrica diminui até um valor mínimo e então aumenta, quando a quantidade destas fases é aumentada.

Esta discrepância pode ser entendida considerando que o parâmetro importante para a constante dielétrica e fator de perda é a composição de cada fase e não só a relação entre suas quantidades. Como a composição de cada fase é dependente da composição das matérias primas que, por sua vez são intrínsecas de cada região, é natural que não exista uma coerência entre os resultados da literatura com relação ao comportamento geral da relação entre microestrutura e propriedades dielétricas.

\section{EFEITO DE ADITIVOS NA MICROESTRUTURA DE PORCELANAS}

A literatura apresenta diversos resultados sobre o estudo da influência de aditivos no desenvolvimento da microestrutura de porcelanas. Os aditivos geralmente são adicionados na forma de óxidos sendo $\mathrm{Fe}_{2} \mathrm{O}_{3}, \mathrm{TiO}_{2}, \mathrm{CaO}, \mathrm{MgO}, \mathrm{Cr}_{2} \mathrm{O}_{3}, \mathrm{Na}_{2} \mathrm{O}$ e $\mathrm{K}_{2} \mathrm{O}$, os mais estudados.

Johnson e Pask [44] estudaram o efeito da adição de óxidos presentes na caulinita $\left(\mathrm{TiO}_{2}, \mathrm{Fe}_{2} \mathrm{O}_{3}, \mathrm{Na}_{2} \mathrm{O}\right.$ e $\left.\mathrm{K}_{2} \mathrm{O}\right)$ em misturas equivalentes de $\mathrm{Al}_{2} \mathrm{O}_{3}-\mathrm{SiO}_{2}$. Estudaram também o efeito da adição de $\mathrm{Fe}_{2} \mathrm{O}_{3}$ e CaO tanto na caulinita quanto na mistura de $\mathrm{Al}_{2} \mathrm{O}_{3}-\mathrm{SiO}_{2}$. Determinaram que na mistura de $\mathrm{Al}_{2} \mathrm{O}_{3}-\mathrm{SiO}_{2}$ a adição de $\mathrm{TiO}_{2}, \mathrm{Fe}_{2} \mathrm{O}_{3}$ e $\mathrm{CaO}$ diminui a viscosidade da fase líquida formada durante a sinterização aumentando a difusividade dos íons com conseqüente aumento da taxa de crescimento dos cristais de mulita. $\mathrm{O} \mathrm{Fe}_{2} \mathrm{O}_{3}$ e $\mathrm{CaO}$ apresentaram um efeito mais pronunciado no crescimento dos cristais da mulita. Este efeito aparece, mas de maneira menos pronunciado quando $\mathrm{o} \mathrm{Fe}_{2} \mathrm{O}_{3}$ e o $\mathrm{CaO}$ são adicionados à caulinita. A adição de 5,7\% em peso de $\mathrm{Fe}_{2} \mathrm{O}_{3}$ fez com que a forma dos cristais de mulita formados a partir da mistura $\mathrm{Al}_{2} \mathrm{O}_{3}$ $\mathrm{SiO}_{2}$ fossem similares à encontrada para os cristais formados a partir da caulinita. Isto foi atribuído a uma significante quantidade de íons $\mathrm{Fe}^{3+}$ em solução sólida na mulita, de $6 \%$ a
$12 \%$ para temperaturas de $1300{ }^{\circ} \mathrm{C}$ a $1400{ }^{\circ} \mathrm{C}$.

Tkalsec et al. [45] estudaram a introdução de $\mathrm{BaCO}_{3}$ em porcelanas contendo $20 \%$ de alumina e mostraram que a presença de $4 \%$ de íons $\mathrm{Ba}^{2+}$ aumenta de $102 \mathrm{MPa}$ para $114 \mathrm{MPa}$ o módulo de ruptura destas porcelanas. Este aumento é atribuído à variação na microestrutura e na composição da fase vítrea. Foi observado que para composições com até $2 \%$ de $\mathrm{BaCO}_{3}$ não há alterações no valor da rigidez dielétrica, sendo que uma pequena redução foi encontrada para composições com $4 \%$ de $\mathrm{BaCO}_{3}$. Mostraram também que a presença de íons $\mathrm{Ba}^{2+}$ reduz o tamanho e a quantidade de cristais de mulita e favorecem a dissolução da alumina.

Pradonovic e Zivanovic [46] determinaram que a introdução de íons $\mathrm{Cr}^{3+}$ em porcelanas elétricas com $45 \%$ em peso de alumina promoveu a formação prematura da fase líquida durante a sinterização aumentando a dissolução da alumina e formando fase vítrea rica em alumínio, a qual possui resistência mecânica maior que fase vítrea rica em silício.

Tasic [47] estudou o efeito da adição de $\mathrm{Cr}_{2} \mathrm{O}_{3}, \mathrm{MnO}_{2}$ e $\mathrm{ZnO}$ em uma porcelana com composição de $45 \%$ de alumina, $9 \%$ de caulim, $25 \%$ de argila e de $21 \%$ a $19,5 \%$ de feldspato e observou redução de $60{ }^{\circ} \mathrm{C}$ a temperatura de sinterização, aumento da quantidade de mulita primária de $2 \%$ a $3 \%$, inibição do crescimento dos cristais de mulita secundária, aumento da densidade e redução da porosidade das peças. Entre todos os aditivos o $\mathrm{Cr}_{2} \mathrm{O}_{3}$ foi o que promoveu maior incremento nas propriedades mecânicas

Chahudhuri e Sarkar [48] estudaram uma porcelana com composição $63 \%$ de argila, $12 \%$ de quartzo e $25 \%$ de feldspato com e sem a presença de aditivos $\left(\mathrm{Fe}_{2} \mathrm{O}_{3}, \mathrm{TiO}_{2}, \mathrm{Cr}_{2} \mathrm{O}_{3}, \mathrm{~V}_{2} \mathrm{O}_{5} \mathrm{e}\right.$ $\mathrm{Nb}_{2} \mathrm{O}_{5}$ ) e mostraram que a presença destes óxidos torna a fase vítrea propensa à nucleação e cristalização de cristobalita após tratamento térmico, além de aumentar a quantidade de mulita, reduzindo a quantidade de fase vítrea. A presença de $2 \% \mathrm{em}$ peso de $\mathrm{Nb}_{2} \mathrm{O}_{5}$, aumentou a quantidade de mulita e estimulou o surgimento de grande quantidade de cristobalita $(24,8 \%)$, mesmo sem tratamento térmico.

\section{SUMÁRIO}

Porcelanas elétricas são fabricadas a partir de matériasprimas naturais, basicamente argila, feldspato e quartzo ou alumina. A microestrutura é formada por fases cristalinas dispersas em uma matriz vítrea. A presença de alguns íons altera a relação dessas fases e, por conseqüência, altera as propriedades elétricas e mecânicas. Esses íons podem estar presentes na composição de forma intencional, neste caso chamados aditivos, ou de forma involuntária, neste caso chamados impurezas, introduzidos via matéria prima. Portanto, controle rigoroso das matérias primas é necessário para viabilizar o controle sobre a microestrutura e propriedades.

\section{AGRADECIMENTOS}

À CAPES pela bolsa PICDT de um dos autores (ALC). 


\section{REFERÊNCIAS}

[1] W. M. Carty, J. Am. Ceram. Soc. 81, 1 (1998) 3-20.

[2] P. Vincenzini, Fundamentals of Ceramic Engineering, Elsevier Applied Science Ltd., New York, (1991) p. 27-50.

[3] C. R. Austin, H. Z. Schofield, N. L. Haldy, J. Am. Ceram. Soc 29, 12 (1946) 341-354.

[4] J. Liebermann, W. Schulle, Am. Ceram. Soc. Bull. 81, 2 (2002) 33-38.

[5] J. Liebermann, Am. Ceram. Soc. Bull. 82, 2 (2003) 39-46. [6] A. K. Ismailov, Glass and Ceramics 38, 7-8 (19981) 363364.

[7] S. Villegas-Palacio, D. R. Dinger, Am. Ceram. Soc. Bull. 75, 7 (1996) 71-76.

[8] S. Villegas-Palacio, D. R. Dinger, Am. Ceram. Soc. Bull. 75, 9 (1996) 79-84.

[9] Y. Iqbal, W. E. Lee, J. Am. Ceram. Soc. 83, 12 (2000) 3121-3127.

[10] K. J. D. Mackenzie, I. W. M. Bronw, R. H. Meinhold, M. E. Bowden, J. Am. Ceram. Soc. 68, 6 (1985) 293-97.

[11] A. K. Chakraborty, D. K. Ghosh, J. Am. Ceram. Soc. 61, 3-4 (1978) 170-173.

[12] K. Srikrishna, G. Thomas, R. Martinez, M. P. Corral, S. Aza, J. S. Moya, J. Mater. Sci. 25 (1990) 607-612.

[13] B. Sonuparlak, M. Sarikaya, I. A. Aksay, J. Am. Ceram. Soc. 70, 11 (1987) 837-42.

[14] M. J. Hyatt, N. P. Bansal, J. Am. Ceram. Soc. 25 (1990) 2815-2821.

[15] T. Takei, Y. Kameshima, A. Yasumori, K. Okada, N. Kumada, N. Kinomura, J. Non-Cryst. Sol. 282 (2001) 265-277. [16] T. Takei, Y. Kameshima, A. Yasumori, K. Okada, J. Eur. Ceram. Soc. 21 (2001) 2487-2493.

[17] A. L. Chinelatto, "Efeito de Terras Raras no desenvolvimento da Microestrutura e nas Propriedades de Porcelanas Aluminosas", Tese (Doutorado em Ciência e Engenharia de Materiais), Programa de Pós-Graduação em Ciência e Engenharia de Materiais, Universidade Federal de S. Carlos, S. Carlos (2002) 174f.

[18] Y. Iqbal, W. E. Lee, J. Am. Ceram. Soc.82, 12 (1999) 3584-3590.

[19] Y. Iqbal, W. E. Lee, J. Am. Ceram. Soc. 83, 12 (2000) 3121-3127.

[20]. W. E. Lee, Y. Iqbal, J. Eur. Ceram. Soc. 21 (2001) 2583 2586.

[21] R. C. Buchanan, Ceramic Materials for Electronics Processing, Properties, and Applications, Marcel Dekker, New York (1986)

[22] F. H. Norton, Cerámica Fina - Tecnología y Aplicaciones, $3^{a}$ ed., Ediciones Omega S. A., Barcelona (1988) p. 384-404.
[23] R. Biensen, G. A. Geyer, K. H. Schüller, Ceram. Forum Int./Ber.DKG. 64, 10 (1987) 376-379.

[24] A. M. Bishai, B. H. F. Al-Khayat, F. A. Awni, Am. Ceram. Soc. Bull. 64, 4 (1985) 598-601.

[25] E. P. Goulart; M. A. P. Jordão, D. D. D. Souza, P. K. Kiyohara, Cerâmica 36, 242 (1990) 32-40.

[26] Y. Kobayashi, O. Ohira, T. Satoh, E. Kato, Br. Ceram. Trans. 93, 2 (1994) 49-52.

[27] I. A. Popova, D. B. Kreimer, Glass Ceram. 47, 3-4 (1990) 90-92.

[28] G. Peña, A. G. Verduch, F. S. Del Rio, Interceram 37, 5 (1988) 65-68.

[29] K. N. Maiti, S. Kumar, Ceram. Int. 18, 6 (1992) 403-412. [30] W. P. Tai, K. Kimura, K. Jinnai, J. Am. Ceram. Soc. 83, 7 (2000) 1658-1662.

[31] S. J. Warshaw, R. Sieder, J. Am. Ceram. Soc. 50 (1967) 337-343.

[32] S. C. Sane, R. L. Cool, J. Am. Ceram. Soc. 34 (1951) 145-151.

[33] J. E. Schroeder, Am. Ceram. Soc. Bull. 57, 5 (1978) 526.

[34] L. Mattyasovszky-Zsolnay, J. Am. Ceram. Soc. 40 (1957) 299-306.

[35] G. N. Maslennikova, R. G Orlova, V. D Beshentsev, E. P. Bogdanis, Glass Ceram. 41, 9-10 (1984) 450-452.

[36] N. Sugiyama, R. Harada, H. Ishida, J. Ceram. Soc. Jpn. Int. Ed. 105, 2 (1997) 134-139.

[37] R. G. Orlova, V. D. Beshentsev, J. K. Moroz, E. P. Bogdanis, Glass Ceram. 45, 11/12 (1988) 466-469.

[38] D. B. Kreimer, T. L. Chistyakova, Glass Ceram. 46, 1112 (1989) 489-491.

[39] J. Liebermann, Am. Ceram. Soc. Bull. 80, 6 (2001) 37-42. [40] J. Liebermann, Am. Ceram. Soc. Bull. 80, 7 (2001) 43-48. [41] F. K. Tadzhiev, R. I. Ismatova, T. T. Abdikhodzhaev, Glass Ceram. 47, 9-10 (1990) 355-357.

[42] S. P. Chaudhuri, P. Sarkar; A. K. Chakraborty, Ceram. Int. 25 (1999) 91-99.

[43] S. P. Chaudhuri, P. Sarkar., Ceram. Int. 26 (2000) 865875.

[44] S. M. Johnson, J. A. Pask, Am. Ceram. Soc. Bull. 61, 8 (1982) 838-842.

[45] E. Tkalsec, D. Pradonovic, Br. Ceram. Trans. J. 84 (1985) 94-98.

[46] D. Pradonovic, B. Zivanovic, Interceram 39, 1 (1990) 41-42.

[47] Z. D. Tasic, J. Mater. Sci. 28 (1993) 5693-5701.

[48] S. P. Chaudhuri, P. Sarkar, J. Eur. Ceram. Soc. 15 (1995)1031-1035.

[49] D. Melzer, F. Müller, K. Timmerman, Ceram. Forum Int./ Ber.DKG 70, 9 (1993) 493-495.

(Rec. 29/09/03, Ac. 05/12/03) 